\title{
Priority Hierarchies in Subject Assignment in English: Cases of Conflation
}

\section{Carolina Rodríguez Juárez University of Las Palmas de Gran Canaria} crodriguez@dfm.ulpgc.es

\begin{abstract}
The theoretical model of Functional (Discourse) Grammar initially developed by Simon C. Dik (1997a/b) stresses the relevance of implicational hierarchies in grammatical operations and claims that these hierarchies, although they present individual properties associated with intrinsic, functional and hierarchical constraints, may interact with each other to the extent that some of them could be grouped and reformulated as a single hierarchy gathering properties of different nature. In this paper, we will explore these cases of conflation within the domain of the grammatical operation of Subject assignment in one particular language, viz. English, and will suggest new cases of overlap between some of these priority features which might be appropriate in a descriptive approach to Subject selection in the English language.
\end{abstract}

\section{Introduction}

The theoretical model of Functional Grammar (henceforth FG) initially developed by Simon C. Dik (1997a; 1997b) and recently improved and turned into a new architecture known as Functional Discourse Grammar (FDG) by Kees Hengeveld and Lachlan Mackenzie (Hengeveld, 2004a/b; Hengeveld and Mackenzie, [to appear]), seeks to explain the reflection of the structure of natural languages as regards their main purpose, communication. This functional approach claims that the different linguistic constructions which have been registered in natural languages are the result of the application of different operations to various term positions within a predication. One of these grammatical 
operations is the assignment of syntactic functions to different terms of a predication. Thus, many languages, among which English is found, give the speaker the possibility of describing the same State of Affairs (SoA hereafter) from different viewpoints depending on the term within the same predication to which Subject function has been assigned.

The alternative possibilities of Subject assignment will lead us on the one hand to active constructions provided that this function has been assigned to a first argument ((A1) a term position to which any of the following semantic functions has been assigned: Agent, Positioner, Force, Processed [Exp], Zero [Exp])), and on the other hand to passive constructions depending on whether Subject function has been assigned to a non-first argument, viz. to a second argument (A2) with the semantic functions of Goal, Recipient, Location, Direction, Origin or Reference, to a third argument (A3) with the semantic functions of Recipient, Location, Direction, Origin or Reference in the case of ditransitive predications, or less frequently to first-level satellites such as Beneficiary and under restricted circumstances to some second-level satellites such as Temporality or Location. ${ }^{1}$

The different options of Subject assignment therefore allow the basic perspective designated by the predicate (from the first argument, to the second (A2) and from this to the third (A3)) to be modified and reversed as a result of the assignment of Subject function to a term position other than the $\mathrm{A} 1$ in the predication. This is the reason why in FG Subject is described as a pointer which indicates the entity from which the SoA has been presented, and hence the suggested expression of perspectival function to refer to this function (Dik, 1997a: 27). Within FDG, syntactic functions are studied within the grammatical component and are regarded as grammatical notions which are placed at the structural (morphosyntactic) level, becoming operative once the pragmatic (interpersonal level) and semantic (representational level) functions have been assigned. Expression rules will finally determine the term which should be assigned Subject or Object function. Thus, there has been a change from FG to FDG in the sense that syntactic functions are no longer defined as purely perspectival notions which show the viewpoint adopted by a speaker when presenting a particular SoA (Dik 1997a: 251), but are rather defined as grammatical notions which are the result of pragmatic and semantic choices at higher levels (Hengelveld, 2004b: 373-374).

The degree of accessibility of terms to Subject function is conditioned and restricted by intrinsic and functional properties which are presented in the form of implicational hierarchies which predict the priority of some term positions over others in having access to Subject. The predictions established by some of these priority hierarchies may overlap with others as has been pointed out by Dik, who claims for example that the Person and Definiteness Hierarchies may conflate into just one hierarchy which includes both kinds of intrinsic properties (1997a: 37). Following up on this idea, my hypothesis is that further examples of conflation between hierarchies may be proposed within the domain of the grammatical operation of Subject assignment and with particular reference to the English language.

The data analysed in order to check the interaction between priority hierarchies have been gathered from the $L O B$ corpus of written British English and include a corpus sample 
of 2,313 examples. This study has been limited to those constructions which allow the possibility of alternative Subject function assignment in the English language, i.e. to predications with more than one argument which allow both the presentation of the SoA from the perspective of the first argument as well as from the standpoint of a non-first argument, which means that the data is exclusively made up of transitive active (Subject selection: first argument) and passive constructions (Subject selection: non-first argument). Both the active and passive groups which constitute the corpus are made up of main clauses as well as adverbial, relative and nominal subordinate clauses which have been selected in terms of factors such as polarity, mood and finiteness. ${ }^{2}$

The remainder of this paper is structured as follows. In the following section I will provide a general functional account of the notion of accessibility which is directly connected with the grammatical operation of Subject assignment and will present the characteristics and motivations which are linked to the concept of implicational hierarchies. In section 3, I will first deal with the cases of conflation of hierarchies which have been presented in the literature observing their implication for Subject selection in English, and will then suggest other instances of overlap which are directly relevant in Subject assignment in English. The last section of this paper presents the concluding remarks as far as the interaction of priority hierarchies in Subject assignment for the English language is concerned.

\section{Subject assignment: Accessibility and hierarchies}

The various alternatives of Subject assignment can be explained in relation to the degree of accessibility of some terms over others. The concept of accessibility which Dik borrows from Keenan $(1976,1987)$ and Keenan and Comrie (1977) is thus central in the analysis of the grammatical operation of Subject assignment and is defined as "the capacity of a term position to be the target of some grammatical operation. A term position $T$ to which an operation $O$ can be applied is accessible to $O$; otherwise it is inaccessible to $O$ " (Dik, 1997b: 357). This functional approach claims that the degree of accessibility of term positions is conditioned and restricted by hierarchical, functional and intrinsic properties which reflect semantic, pragmatic and cognitive priorities which can be gathered in linear sequences which predict the degree of accessibility of term positions to grammatical operations. These sequences present implicational universals which are organised into hierarchies which describe priorities that seem to have both intralinguistic and interlinguistic validity and that have been claimed to impinge on grammatical operations such as Subject assignment (Dik, 1997a: 279; Dik, 1997b: 359-361).

A hierarchy is conceived in FG as "a sequence of properties, claimed to be of absolute or statistical validity, such that a preceding property can occur without the following properties but not the other way around" (Dik, 1997a: 31). Hierarchies are typologically relevant and typify on the one hand the types of linguistic patterns which may be found across languages and on the other those aspects which differentiate them as regards the 
linguistic subdomain to which the hierarchy has been applied. The relevance of implicational hierarchies for the study of natural languages lies in the fact that they reflect both cognitive aspects which are determined culturally as well as psychologically, and pragmatic aspects which are associated with the deictic centre of the speaker, that is, with what is more familiar and closer to the speaker's pragmatic information. Thus, and according to the predictions established by these hierarchies, that information which is closer to the speaker will be placed first in the linear order of the constituents of a predication. Besides, these hierarchies give information about the frequency of use of certain grammatical constructions in natural languages, rather than information about the possibility or impossibility of using such constructions (Dik, 1997a: 36).

Different hierarchies have been proposed as relevant in the various grammatical processes, and in the case of the operation of Subject assignment the following group of priority hierarchies which show intrinsic constraints seem to directly influence the chance for a term to be assigned Subject (and also Object) function: the Definiteness Hierarchy (definite > other specific > non-specific), the Person Hierarchy (first person/ second person $>$ third person), the Number Hierarchy (singular number $>$ plural number), the Animacy Hierarchy (human $>$ other animate $>$ inanimate force ${ }^{3}>$ other inanimate), the Concreteness Hierarchy (concrete entities $>$ abstract entities), the Entity Hierarchy (firstorder entities $>$ higher-order entities) and the Predication Hierarchy (terms from the same predication $>$ terms from a subordinate predication) (Dik, 1997a: 279). I have proposed a further hierarchy to the study of Subject assignment, the Term Hierarchy, which predicts the accessibility of term positions taking into account the internal structural complexity of the term and which predicts that simple or primary terms will be more accessible to Subject than complex terms realized by finite embedded constructions, which in turn will be more accessible than non-finite complex constructions (Rodríguez Juárez, 2003: 391-409; Rodríguez Juárez, [to appear]).

On the other hand, functional semantic constraints also condition the accessibility of terms to Subject and have been gathered in the Semantic Function Hierarchy (Arg-1 > Goal $>$ Recipient $>$ Beneficiary $>$ Instrument $>$ Location $>$ Temporality) which predicts that terms which carry any of the semantic functions grouped under the first argument (Agent, Positioner, Force, Processed [Experiencier], Zero [Experiencer]) will be the most accessible to Subject, followed in frequency and in level of difficulty by terms carrying the semantic functions Goal, Recipient, etc (Dik, 1997a: 266).

Thus, and following the priorities established by the different hierarchies, a predication frame like the one exemplified in (1) which indicates that a predicate hit establishes a relation between two entities represented by two arguments which carry the semantic functions of Agent and Goal respectively, could be expressed by two different linguistic expressions (examples (2) and (3)) (Dik, 1997a: 252):

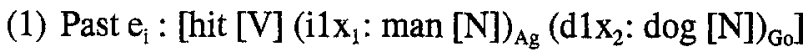

(2) The dog was hit by a man.

Past $\mathrm{e}_{\mathrm{i}}$ : [hit [V] $\left.\left(\mathrm{ilx}_{1}: \operatorname{man}[\mathrm{N}]\right)_{\mathrm{Ag}}\left(\mathrm{d}_{1} \mathrm{x}_{2}: \operatorname{dog}[\mathrm{N}]\right)_{\mathrm{GoSub}}\right]$ 
(3) A man hit the dog.

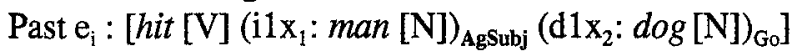

If these two sentences are analysed in terms of the priority established by the Definiteness Hierarchy, the preference of example (2) over the third one would be expected since this hierarchy predicts the higher degree of accessibility of definite terms to Subject function, as can be seen by the fact that Subject function has been assigned to the definite term (the $d o g$ ). On the other hand, if Subject assignment is analysed in terms of the prediction established by the Animacy Hierarchy, sentence (3) would be expected since a term with a human referent ( $m a n$ ) has been more accessible to Subject than the non-human animate referent $(\operatorname{dog})$. This simple example shows how the different hierarchies compete one against the others at the same time within a predication so that their prediction is fulfilled and how very often one wins out over the others. ${ }^{4}$

\section{Cases of interaction between hierarchies}

Different authors have highlighted the interaction existing between some of the intrinsic properties presented in implicational hierarchies. In this section, I will first present the interaction obtaining between the Person Hierarchy and the Definiteness Hierarchy as proposed by Dik among other authors, and will then revise the examples found in the literature as far as the overlap between the Person and the Animacy Hierarchies is concerned. The appropriateness of theses cases of interaction will be explored in the context of a descriptive study of Subject selection in English. In the final part of this section, two other cases of conflation which seem to be relevant for Subject assignment in English will be suggested.

Dik points out that the Person Hierarchy is linked to the Definiteness Hierarchy in the sense that the first and second persons must be "necessarily definite" (1997a: 37), and as a result these two hierarchies may be combined into The Person / Definiteness Hierarchy:

\section{$\{1,2\}>3$ definite $>$ other specific $>$ non-specific}

In fact, the prediction established by this hierarchy that the first person and the second person must be necessarily definite was verified in $100 \%$ of the examples analysed in the data obtained from the $L O B$ corpus. As a result, placing the speech act participants in the left extreme of this hierarchy predicting their priority over the third definite person in the accessibility of term positions to Subject assignment seems appropriate. The Person / Definiteness Hierarchy also predicts that a third-person definite term takes priority over a third-person indefinite term in Subject assignment, as has also been corroborated in the analysis of our data in the case of both active and passive constructions. Table 1 breaks down a complete analysis of the various linguistic patterns registered in the data as far as the relationship between third-persons (represented by " $t$ " in the table) and definiteness 
(" $d$ " for definite and " $i$ " for terms which present other forms of specification or which are indefinite non-specific) is concerned. The different linguistic patterns gather information about the intrinsic properties attributable to the two terms within the predication which could potentially be assigned Subject function separated by the symbol " $>$ ", which indicates that the term preceding it has been assigned Subject function and as a consequence has been more accessible to such function than the term following it. These terms are the Subject and Object of active constructions and the Subject and the by-phrase of passive examples.

\begin{tabular}{|l|c|c|c|c|c|c|}
\hline $\mathrm{t}>\mathrm{t}$ & \multicolumn{2}{|c|}{ Passives } & \multicolumn{2}{c|}{ Actives } & \multicolumn{2}{c|}{ Total corpus (1765) } \\
\hline & No. & $\%$ & No. & $\%$ & No. & $\%$ \\
\hline $\mathrm{d}>\mathrm{d}$ & 100 & $14.5 \%$ & 468 & $43.6 \%$ & 568 & $32.2 \%$ \\
\hline $\mathrm{i}>\mathrm{i}$ & 180 & $26.0 \%$ & 78 & $7.3 \%$ & 258 & $14.6 \%$ \\
\hline $\mathrm{d}>\mathrm{i}$ & 402 & $58.2 \%$ & 502 & $46.7 \%$ & 904 & $51.2 \%$ \\
\hline $\mathrm{i}>\mathrm{d}$ & 9 & $1.3 \%$ & 26 & $2.4 \%$ & 35 & $2.0 \%$ \\
\hline Total & 691 & $100 \%$ & 1074 & $100 \%$ & 1765 & $100 \%$ \\
\hline
\end{tabular}

Table 1. Relationship between the linguistic pattern $t>t$ and the Definiteness Hierarchy

Another example of overlap between hierarchies mentioned by Dik also includes the Person Hierarchy, although in this case the properties first-person and second-person, attributable to the participants of the speech act, are closely related to the property human, which implies that the Animacy and Person Hierarchies could conflate into the so-called The Person / Animacy Hierarchy, in the sense that the first and second persons "necessarily refer to human entities" (1997a: 37):

\section{$\{1,2\}>3$ human $>$ animate $>$ inanimate force $>$ other inanimate}

Similarly, Siewierska presents another case of conflation of properties attributable to different hierarchies by including within the Person Hierarchy, animacy and abstraction features (1991: 106), which is exactly parallel to the one proposed by Allan (1987:57) and which is called The Personal Hierarchy: $1^{\text {st }} p>2^{\text {nd }} p>3^{\text {rd }} p$ human $>$ higher animals $>$ other organisms $>$ inorganic matter $>$ abstracts. Allen and Frantz (1984: 305) in the same line suggest a hierarchy "in which first and second person outrank animate third person which in turns outranks inanimate third person." Hawkinson and Hyman (1974: 169) on the other hand present a hierarchy called The Animacy Hierarchy which also includes properties associated with the Person Hierarchy but they abbreviate the animacy features to three big groups: humans, animals and inanimate entities: $1^{\text {st }} / 2^{\text {nd }}>$ human $-3^{\text {rd }}>$ animal $-3^{\text {rd }}>$ inanimate- $3^{\text {rd }}$.

Nevertheless, the relevance of the Person / Definiteness hierarchy as regards Subject assignment in the English language may be questioned since there are examples where the prediction established by one of the two hierarchies belonging to the Person / Definiteness hierarchy is fulfilled whereas the other is not. Let's take a concrete example to illustrate the 
inoperativeness of the Person / Definiteness hierarchy in Subject assignment. It might be the case that the Person hierarchy has not been fulfilled because a third-person term has had access to Subject instead of one of the speech act participants (third $>$ first/second); however, this circumstance does not necessarily entail that the definiteness prediction has also been unfulfilled, as could be observed in the analysis of the data where $31.8 \%$ of the passive examples and $52.2 \%$ of the active sentences correspond to the instances in which the two terms analysed share the same property, that is to say, they are both either definite (example 4) or indefinite (example 5).

(4) The egg-cases, which my good friend, Mr J. Moncrieff (third person / definite), export manager of the well-known American seed firm, Atlee Burpee and Co., kindly sent me (first person / definite) were of the Arizona species, ... (1169/E07-82 $)^{5}$

(5) Interesting attempts (third person / indefinite) have been made by various writers (third person / indefinite). (310/J18-14)

In fact, the examples in the global corpus in which both terms are either definite or indefinite outweigh the cases in which definite and indefinite terms compete for Subject assignment, and are as a result not really relevant for the study of the intrinsic property of definiteness. What is more, the prediction of both hierarchies is only violated in 23 of the sentences which make up the corpus of active and passive constructions, of which 6 instances are examples of active sentences (example 6) and 17 are examples of passive constructions (example 7).

(6) It may be pure coincidence, but a number of people (third person / indefinite) have asked me (first person / definite) "whatever makes you interested in local government?" (1199/F16-146)

(7) Extra money (third person / indefinite) could be usefully spent [by us (first person / definite)] on it, ... (731/L15-119)

Likewise, many of the examples in which the Definiteness hierarchy has been violated are irrelevant from the point of view of the Person hierarchy because the two terms which could potentially be assigned Subject function are third-persons ( 7 examples in the case of passive constructions (example 8 ) and 41 examples in the case of active sentences (example 9)), and should never be interpreted as cases in which the Person hierarchy has been violated.

(8) Nobles and others (third person / indefinite) whose status is dependent on hereditary privilege rather than personal merit (...) were swiftly followed by the smaller fry (third person / definite) who saw in the lit de parade an easy and comfortable method of establishing their social superiority. (576/F06-139) 
(9) No one (third person / indefinite) can blame Harold Macmillan (third person / definite) for trying to reach the elusive goal. (1123/B20-193)

Therefore, in spite of the fact that it is evident that the Definiteness and Person hierarchies present characteristics which could overlap, it seems that the conflation of both properties into just one hierarchy is not appropriate for the study of the grammatical operation of Subject assignment in English, and as a result for the purposes of this our research it has been agreed that the relevant term positions should be analysed in virtue of each of the individual properties separately.

On the other hand, I completely go along with the proposal that the Person and Animacy Hierarchies may conflate into just one hierarchy since there exists a clear overlap between the properties "first/second person" and the feature "human", and as a consequence it could correctly be claimed that the resulting hierarchy (The Person / Animacy Hierarchy) is relevant for the analysis of Subject assignment in English. However, as has already been mentioned, for the purposes of this research the Person Hierarchy should be studied and analysed as a single hierarchy.

Nevertheless, the results obtained from the same research have provided evidence that has allowed me to conclude that new cases of interaction of hierarchies could be suggested in relation to Subject assignment in the English language. The first example of conflation between hierarchies which will be proposed is the one which includes the Concreteness Hierarchy and the Entity Hierarchy. Let's in the first place present the two hierarchies separately and then show the way they could interact.

The Entity Hierarchy which according to Dik could be represented as first-order entities $>$ higher-order entities (1997a: 279) predicts that first-order entities $\left(\mathrm{x}_{\mathrm{i}}\right)$, which refer to physical objects, individuals and places located in space, are more accessible than second-order entities $\left(e_{i}\right)$, which make reference to SoAs, and these in turn are more accessible than those which describe a possible fact $\left(\mathrm{X}_{\mathrm{i}}\right.$ : third-order entities), which will at the same time be more accessible than fourth-order entities $\left(E_{i}\right)$, which are associated with speech acts. However, the figures obtained from the analysis of the data reveals that the prediction established by this hierarchy is not completely accurate in the sense that it does not include zero-order entities, which make reference to properties or relations typically associated with first-order entities and which may also be assigned Subject function (example 10$)$, although in a lower percentage $(0.3 \%)$.

... and its bow front gave it [a chest of drawers] an elegance which pleased them both. (1504/P16-115)

Therefore, I propose a reformulation of the Entity Hierarchy presented by Dik and suggest the following sequential ordering in which first-order entities are placed at the left extreme of the hierarchy (which are in fact the ones which are more often assigned Subject function), and all the other entities, including zero-order entities, are placed at the right side of the hierarchy, without specifying any priority among them, since $98.8 \%$ of the examples 
analysed include first-order entities competing with any other-order entity in order to be assigned Subject function. Nevertheless, it was possible to predict different degrees of accessibility among the non-first-order entities when these compete with a first-order entity, second-order entities being the ones which more frequently have access to Subject, followed by third-order entities and fourth-order entities, and all these being in turn more accessible than zero-order entities. Thus, the reformulation of this hierarchy could be schematically represented as first-order entities $>$ other-order entities (or non-first order entities).

The Concreteness Hierarchy, on the other hand, predicts that those terms whose referents present concrete features which may be perceived through the senses will be more accessible than those terms with abstract reference which either represent concepts which exist in our minds or make reference to entities which denote events, states, qualities or activities (Quirk et al., 1985: 5.3).

These two types of intrinsic constraints overlap in the sense that first-order entities must necessarily be concrete (example 11), as can be seen by the fact that they can be located in space, whereas non-first-order entities, which denote either properties or relations (zeroorder entities, example 10), actions, processes, states and positions (second-order entities, example 12), possible facts (third-order entities, examples 13 and 14), and speech acts (fourth-order entities, examples 15 and 16 ) must necessarily be abstract. ${ }^{6}$

(11) A citizen of the U.S was last week walking down Oxford-street when he (first-order entity) was seized by $a$ total stranger (first-order entity)... (55/B05-14).

(12) General de Gaulle's official welcome (second-order entity) last week to Britain's moves towards the six was taken as a friendly gesture in Whitehall. (8/A02-09)

(13) The government believe that a plan on these lines would not begin to meet the needs of the situation (third-order entity). (2071/H11-76)

(14) It is felt that the above correction is not entirely satisfactory (third-order entity) as it is based on fixed wing theory. (297/J73-133)

(15) ... and the answer (fourth-order entity), again, is best given in personal terms, ... (618/G59-46)

(16) It was stated that the ministry had no power to return the property to the council at no cost (fourth-order entity). (34/A11-135)

In the light of the alleged direct relationship existing between entity types and the feature of abstraction, the two hierarchies presenting such intrinsic properties could conflate in what I have come to call the Entity-Concreteness Hierarchy which could be represented as first-order concrete entities $>$ other-order abstract entities (or first-order concrete entities 
$>$ non-first-order abstract entities).

As for the validity of the Entity-Concreteness Hierarchy, the results obtained from the study of the relevant data show that the hierarchy is obeyed in different degrees depending on the type of argument which is in Subject position. In the cases in which a first argument has had access to Subject (active constructions), the hierarchy is obeyed in $97.1 \%$ of the examples; however, if a non-first argument has been assigned Subject function (passive constructions) this percentage is reduced to $7.9 \%$.

A second type of interaction between priority hierarchies which was observed in the analysis of our data is closer to the overlap suggested by Siewierska (1991: 106) and Allan (1987: 57) mentioned before, and is related to the intrinsic properties of animacy, concreteness and entities. The interaction between these hierarchies is justified in the sense that term positions which have the animacy property of human, non-human animate or inanimate force are necessarily first-order entities and therefore concrete entities, whereas inanimate terms may be either concrete first-order entities or other-order entities, which are necessarily abstract and less accessible to Subject function than concrete first-order entities:

Hum. $>$ no-hum. animate $>$ inanimate force $>\quad$ inanimate $1^{\text {st }}$-order, concrete $\quad 1^{\text {st }}$-order, concr. $>$ other-order, abstr.

The conflation of these properties may be represented in a single hierarchy, the Entity (Concreteness) - Animacy Hierarchy: human $>$ non-human animate $>$ inanimate-force $>1^{\text {st }}$-order inanimate $>$ other-orderinanimate. The degree of incidence of this hierarchy in comparison with those which gather structural and semantic properties is not so determinant in Subject assignment and is highly dependent on the kind of term which has had access to Subject. If Subject assignment has been assigned to an A1 (active constructions), the percentage of fulfilment of the hierarchy is very high $(96.8 \%)$, whereas in the marked cases in which a non-first argument has been assigned Subject function (passive sentences) the percentage of fulfilment is reduced to $6.7 \%$.

\section{Conclusion}

The different term positions which constitute a predication present functional, hierarchical and intrinsic properties which condition the accessibility of such terms to grammatical operations like Subject assignment. It has been the main concern of this research to study the degree of accessibility of term positions to Subject function in the English language and to observe the instances of overlap obtaining between some priority hierarchies which constrain their accessibility in virtue of structural, semantic and referential properties.

In the first place, and following various linguists' proposals, I mentioned one case of conflation between those hierarchies which present grammatical constraints associated with term operators expressing distinctions in the semantic domain of definiteness and person. 
These two types of intrinsic properties could be unified in a single hierarchy (the Person / Definiteness Hierarchy) due to the overlap between the features "first/second persons" and "definite". Similarly, the Person / Animacy Hierarchy was presented as an example of interaction in which the semantic feature of "person" was associated with referential aspects gathered in the Animacy Hierarchy. Nevertheless, for the purposes of this research the stance that the Person, Animacy, and Definiteness Hierarchies should be analysed separately was adopted since in the study of Subject assignment in English the violation of the prediction of one of the hierarchies does not necessarily presuppose the violation of the other.

As for the instances of interaction between hierarchies which have been suggested in this paper, they present features which describe Subject accessibility in the English language in terms of the referential properties of the term. In the first place, I presented the conflation of the Entity Hierarchy and the Concreteness Hierarchy, and supported their overlap by proving that first-order entities must necessarily be concrete whereas otherorder entities are abstract. In this sense, the Entity-Concreteness Hierarchy was formulated as follows: first-order concrete entities $>$ other-order abstract entities. Another case of interaction was observed as regards the Animacy Hierarchy. The referential properties associated with this priority hierarchy seem to be directly associated with the features gathered in the Entity-Concreteness Hierarchy in the sense that first-order concrete entities are related to humans, non-human animates or inanimate forces, whereas inanimate entities may be either first-order concrete entities or other-order abstract entities. Thus, the Entity (Concreteness) - Animacy Hierarchy, which includes three different types of intrinsic properties, was suggested: human $>$ non-human animate $>$ inanimate-force $>1^{\text {st }}$-order inanimate $>$ other-order inanimate.

With this proposal, I have tried to contribute to enhancing the position adopted by the functional appreach as regards Subject assignment by providing a multi-dimensional description of Subject selection with regard to the overlap of the referential properties of potential Subjects in the English language.

\section{Notes}

1. Within the context of FG, arguments are defined as "terms which are required by some predication in order to form a complete nuclear predication. They are essential to the integrity of the SoA designated by the predicate" (Dik, 1997a: 86-87) Satellites, on the other hand, are also expressions which can be used to refer to entities but which are not required by the predicate and which provide optional further information pertaining to the SoA (Dik, 1997a: 87).

2. For a full description of the selection processes and the statistical methods used in the gathering of the relevant data, see Rodríguez Juárez (2003: 172-209).

3. Inanimate forces refer to entities such as "wind, storm, rain", etc. (Dik, 1997a: 35) and are classified in the "EuroWordNet top-ontology" as concrete first-order entities (EuroWordNet Top Ontology. Ed. Piek Vossen. Sept. 2001. Department of Computational Linguistics. U of Amsterdam. 17 June 2005 
< http://www.illc.uva.nl/EuroWordNet/corebcs/ewnTopOntology.html\#_Toc419884306>).

4. For an account of the dominance of some hierarchies over others see Rodríguez Juárez (2003: 413-438) where I have suggested a multi-dimensional description of Subject assignment in English which shows different degrees of incidence of some priorities over others.

5. The references which come at the end of each example indicate (i) the number that I have assigned to that example in the total corpus (310), (ii) the type of text from which the example was taken represented by means of a capital letter (A: press: reportage; B: press: editorial; C: press: reviews; D: religion; E: skills, trades, and hobbies; F: popular lore; G: belles lettres, biography, essays; H: miscellaneous: government documents, foundation reports, industry reports, college catalogue, industry house organ; J: learned and scientific writings; $\mathrm{K}$ : general fiction; L: mystery and detective fiction; $\mathrm{M}$ : science fiction; $\mathrm{N}$ : adventure and western fiction; $\mathrm{P}$ : romance and love story; R: humour), and (iii) the number and line assigned to the text in the $L O B$ corpus (in this example 07 and 82 respectively).

6. According to Quirk et al. (1985: 5.3) concrete entities are "accessible to the senses, observable, measurable" whereas abstract nouns denote "unitary phenomena (such as events) on the one hand, or states, qualities, activities, etc on the other" (1985: 5.58). The examples of finite and non-finite embedded clauses are also regarded by these authors as having abstract reference: "they [nominal clauses] refer to such abstractions as events, facts, dates and ideas rather than perceptible objects" (1985: 15.2). Thus, in the current study, the examples of finite and non-finite subordinate clauses as Subject, including examples of extraposed Subjects introduced by the anticipatory $i t$ (see examples 14 and 16 in the text), are analysed as indefinite terms which refer to inanimate abstract entities.

\section{Works Cited}

Allan, Keith (1987): "Hierarchies and the choice of left conjuncts (with particular attention to English)". Journal of Linguistics 23: 51-77.

Allen, Barbara J. and Donald G. Frantz (1984): “Advancements and verb agreement in Southern Tiwa". In D. M. Perlmutter and C. R. Rosen (eds.) Studies in Relational Grammar 2. Chicago: University of Chicago Press, 303-314.

Dik, Simon C. (1997a): The Theory of Functional Grammar. Part 1: The Structure of the Clause. Ed. Kees Hengeveld. Berlin and New York: Mouton de Gruyter. (1997b): The Theory of Functional Grammar. Part 2: Complex and Derived Constructions.

Ed. Kees Hengeveld. Berlin and New York: Mouton de Gruyter.

Hawkinson, Annie K. and Larry M. Hyman (1974): "Hierarchies of natural topic in Shona". Studies in African Linguistics 5: 147-170.

Hengeveld, Kees (2004a): "The architecture of a Functional Discourse Grammar". In J. L. Mackenzie and M. Á. Gómez-González (eds.) A New Architecture for Functional Grammar. Berlin and New York: Mouton de Gruyter, 1-21.

. (2004b): "Epilogue". J. L. Mackenzie and $\mathrm{M}^{\mathrm{a}}$ A. Gómez-González (eds.) A New Architecture for Functional Grammar. Berlin and New York: Mouton de Gruyter, 365-378.

Hengeveld, Kees and J. Lachlan Mackenzie (forthcoming): Functional Discourse Grammar. Oxford: Oxford University Press.

Keenan, Edward L (1976): "Towards a universal definition of "subject of" ". In Ch. Li (ed.) Subject and Topic. New York: Academic Press, 303-333. 
. (1987): Universal Grammar: 15 Essays. London: Croom Helm.

Keenan, Edward L. and Bernard Comrie (1977): "Noun phrase accessibility and universal grammar". Linguistic Inquiry 8: 63-99.

Quirk, Randolph, Sidney Greenbaum, Geoffrey Leech, and Jan Svartvik (1985): A Comprehensive Grammar of the English Language. London: Longman.

Rodríguez Juárez, Carolina (2003): La Asignación de la Función Sujeto en inglés: Jerarquías y Prioridades. Unpublished Dissertation. University of La Laguna. . (forthcoming): "A new parameter in the description of Subject assignment: the Term Hierarchy". Atlantis 28(1).

Siewierska, Anna (1991): Functional Grammar. London: Routledge. 